\title{
VARIAÇÃO ESPECTRAL E EFICIÊNCIA DE USO DA RADIAÇÃO FOTOSSINTETICAMENTE ATIVA EM ENSAIO COM GENÓTIPOS DE TRIGO(1)
}

\author{
MAURICIO ALVES MOREIRA ${ }^{(2)}$; BERNARDO FRIEDRICH THEODOR RUDORFF ${ }^{(2)}$; \\ JOÃO CARLOS FELÍCIO ${ }^{(3)}$; JOSÉ GUILHERME DE FREITAS ${ }^{(3)}$; MARCELO DOS SANTOS TARGA ${ }^{4}$
}

\begin{abstract}
RESUMO
Medidas de radiometria de campo são utilizadas para quantificar a variação espectral e a eficiência de uso da radiação solar na produção de fitomassa $\left(\varepsilon_{\mathrm{F}}\right)$, em um experimento com 20 genótipos de trigo, cultivado na área experimental da Universidade de Taubaté, Taubaté, SP, durante o período de maio a outubro de 1999. A cultura foi irrigada em intervalos semanais, visando manter o solo próximo à capacidade de campo. Foram obtidas oito medidas do fator de refletância da cultura do trigo, entre a emergência das plântulas e a maturação fisiológica dos grãos com o radiômetro Spectron SE-590. Essas medidas foram transformadas no índice de vegetação com diferença normalizada (IVDN) e, juntamente com dados de radiação fotossinteticamente ativa (RFA), foram utilizadas no cálculo da RFA absorvida acumulada (RFAAA) ao longo do ciclo da cultura, a fim de estimar os valores de $\varepsilon_{\mathrm{F}}$. Pelo resultado, em $\varepsilon_{\mathrm{F}}$ observou-se significativa variação entre genótipos, a técnica de radiometria de campo é uma ferramenta promissora na identificação de genótipos $\operatorname{com}$ maior $\varepsilon_{\mathrm{F}}$, e ainda, para produção de fitomassa, o aproveitamento da radiação, no processo fotossintético, é bem mais relevante do que a RFAAA.
\end{abstract}

Palavras-chave: trigo, sensoriamento remoto, eficiência de uso da radiação, produção de biomassa.

\section{ABSTRACT \\ SPECTRAL VARIATION AND RADIATION USE EFFICIENCY IN A WHEAT GENOTYPE TRIAL}

Field radiometry measurements are used to quantify the spectral variation and the solar radiation use efficiency of biomass production $\left(\varepsilon_{\mathrm{F}}\right)$, in an experiment with 20 wheat genotypes cultivated at the experimental site of the University of Taubaté in Taubaté, São Paulo State, Brazil, during the period from May to October of 1999. The crop was irrigated on a weekly basis in order to supply water close to soil field capacity. Eight radiometric measurements were taken with the field spectroradiometer Spectron SE-590, from plant emergency through physiological grain maturity. These measurements were transformed into the normalized difference vegetation index (NDVI) that, along with the photosynthetic active radiation (PAR) data, were used to calculate the accumulated absorbed PAR (AAPAR) over the crop growing season in order to estimate the values of $\varepsilon_{\mathrm{F}}$. The result showed that $\varepsilon_{\mathrm{F}}$ presented a significant variation among genotypes and that the field radiometric technique is a promising tool to identify genotypes with high $\varepsilon_{\mathrm{F}}$, and furthermore, for biomass production, the use of solar radiation, in the photosynthetic process, is much more relevant than AAPAR.

Key words: wheat, field radiometry, remote sensing, radiation use efficiency, biomass production

$\left({ }^{1}\right)$ Recebido para publicação em 19 de agosto de 2004 e aceito em 28 de março de 2005 . Trabalho parcialmente financiado pela FAPESP (Processo No 97/11075-7).

$\left({ }^{2}\right)$ Instituto Nacional de Pesquisas Espaciais (INPE), Divisão de Sensoriamento Remoto, Av. dos Astronautas, 1.758, 12227-010 São José dos Campos, SP. E-mail: mauricio@ltid.inpe.br; bernardo@ltid.inpe.br

(3) Instituto Agronômico (IAC), Av. Barão de Itapura, 1.481, 13020-902 Campinas (SP). E-mail:jfelicio@iac.sp.gov.br, jfreitas@iac.sp.gov.br

${ }^{(4)}$ Universidade de Taubaté (UNITAU), Departamento de Ciências Agrárias, Estrada Dr. José Luiz Cembranelli, 5.000, 12081-010 Taubaté (SP). E-mail: m.targa@uol.com.br 


\section{INTRODUÇÃO}

As medidas de radiometria de campo na agricultura são freqüentemente utilizadas em estudos, que relacionam a radiação solar refletida pelas culturas com variáveis agronômicas (DEERING, 1989), tais como: índice de área foliar (IAF), fitomassa, fração da radiação fotossinteticamente ativa absorvida $\left(f_{\mathrm{A}}\right)$ (Asrar et al., 1984). A $f_{\mathrm{A}}$ é uma variável importante em modelos de crescimento de culturas agrícolas (GOUdRIAAN e LAAR, 1994). Pode ser estimada a partir da radiação refletida, em bandas espectrais, das regiões do visível (vermelho; 630 a $690 \mathrm{~nm}$ ) e do infravermelho próximo (780 a $900 \mathrm{~nm}$ ), transformadas matematicamente em índice, tal como o índice de vegetação com diferença normalizada (IVDN) (TUCKER, 1979). Por meio da soma diária do produto entre a $f_{\mathrm{A}}$ e a RFA (radiação fotossinteticamente ativa) incidente no topo da cultura, pode-se estimar a quantidade de RFA absorvida acumulada (RFAAA) ao longo do ciclo da cultura.

A razão entre o valor da fitomassa seca da parte aérea e o valor da RFAAA determina a eficiência de uso da radiação ou eficiência fotoquímica da cultura (DAUGHTRY et al., 1992), variável que auxilia na seleção de genótipos mais eficientes na conversão da energia solar em química. Assim, é importante o conhecimento sobre a variação espectral entre genótipos, ao longo da estação de crescimento e sua relação com variáveis agronômicas.

Hatfield (1981), em estudo sobre a variação espectral de oitenta e dois genótipos de trigo, entre a fase de emborrachamento e ponto de colheita, observou que durante o período de enchimento de grãos ocorreu a maior variabilidade na resposta espectral dos genótipos. Resultado similar foi observado por PINTER JR. et al. (1985), em estudo de radiometria de campo sobre o efeito do ângulo de elevação solar e da arquitetura do dossel na reflectância de seis genótipos de trigo. Os autores concluíram que a reflectância de dosséis de trigo não é apenas função de variáveis agronômicas, como IAF ou fitomassa verde, mas também altamente dependente da geometria de incidência da radiação solar e sua interação com as características da arquitetura dos dosséis vegetativos; verificaram-se nas medidas de reflectância obtidas próximo ao meio dia diferenças máximas entre cultivares para as bandas do infravermelho próximo, enquanto para as bandas do visível as diferenças foram mínimas nesse horário.

No Brasil, não se tem conhecimento do uso das técnicas de sensoriamento remoto e de radiometria de campo, como ferramenta auxiliar na seleção de genótipos, de tal forma que o objetivo desta pesquisa foi avaliar a variação espectral e a eficiência de uso da radiação solar na produção de fitomassa em genótipos de trigo.

\section{MATERIAL E MÉTODOS}

O experimento foi desenvolvido sob condições de campo, na área experimental do Departamento de Ciências Agrárias da Universidade de Taubaté (UNITAU), em Taubaté, São Paulo, com as seguintes coordenadas geográficas: latitude de $22^{\circ} 54^{\prime} \mathrm{S}$, longitude de $45^{\circ} 33^{\prime} \mathrm{W}$ e altitude de $600 \mathrm{~m}$. O clima da região, segundo a classificação de Köppen, é do tipo Cwa (tropical de altitude, com inverno seco e chuvas de verão).

O solo da área em estudo corresponde ao Latossolo (EMBRAPA, 1999). O delineamento estatístico foi em blocos ao acaso, com 20 genótipos de trigo e 4 repetições. A semeadura foi realizada em $1 .^{\circ}$ de maio de 1999, em parcelas com dimensões de $3,0 \mathrm{~m} \times 2,0 \mathrm{~m}$. Utilizou-se espaçamento de $20 \mathrm{~cm}$ entre linhas, com 80 sementes viáveis por metro. A adubação de macro e micronutrientes baseou-se na análise de solo coletada na área de estudo, e de acordo com tabela de recomendação de adubação para a cultura do trigo, para o Estado de São Paulo (IAC, 1999).

A irrigação foi realizada por aspersão, para repor as perdas de água e manter a umidade do solo próximo à capacidade de campo. Para o cálculo da lâmina de água evapotranspirada a ser reposta nos talhões de trigo, empregou-se o manejo de irrigação recomendado pelo Boletim Técnico 167 (IAC, 1999), que tem por base a evaporação do tanque classe A e a necessidade de água da cultura.

O fator de reflectância do dossel do trigo foi obtido semanalmente, ou conforme as condições meteorológicas permitiram, sob condições de céu claro no horário entre 11 e $12 \mathrm{~h}$, desde a emergência das plântulas até a maturação dos grãos. Utilizou-se o radiômetro portátil de campo, modelo SE-590 (Spectron Inc. EUA), que opera em 252 bandas espectrais na faixa de 350 a $1150 \mathrm{~nm}$ do espectro eletromagnético (STEFFEn, 1993). O instrumento foi fixado em mastro de alumínio, a cerca de $3 \mathrm{~m}$ acima da estatura média das plantas, e utilizou-se uma lente com ângulo de visada de $15^{\circ}$, que permitiu obter a radiância do dossel em uma área de $0,5 \mathrm{~m}^{2}$. No início e no fim de cada bloco, mediu-se a radiância refletida de uma placapadrão de sulfato de bário $\left(\mathrm{BaSO}_{4}\right)$, a fim de possibilitar o cálculo do fator de reflectância (FR), que é a razão entre a radiância espectral da amostra (cultura) e da placa-padrão, medida na mesma 
condição de iluminação e observação (Milton, 1987). A partir dos valores espectrais do FR foram computados os valores do FR, em intervalos de comprimento de onda correspondente às bandas TM1 (azul; 450 a $520 \mathrm{~nm}$ ), TM2 (verde; 530 a $610 \mathrm{~nm}$ ), TM3 (vermelho; 630 a $690 \mathrm{~nm}$ ) e TM4 (infravermelho próximo; 780 a $900 \mathrm{~nm}$ ), do sensor TM (Thematic Mapper) a bordo do satélite Landsat, cujas imagens são amplamente utilizadas em estudos agrícolas de sensoriamento remoto. Com os valores das bandas TM3 e TM4 foi computado o índice de vegetação com diferença normalizada (IVDN) (TUCKER, 1979):

$$
\mathrm{IVDN}=\frac{\mathrm{TM} 4-\mathrm{TM} 3}{\mathrm{TM} 4+\mathrm{TM} 3}
$$

Os valores do IVDN, obtidos nas várias datas de coleta de medidas radiométricas, foram empregados na análise de correlação com dados de produção de fitomassa seca total da parte aérea, por ocasião da colheita. As medidas de campo, com coeficientes de correlação (r) mais elevados, foram utilizadas tanto para indicar o estádio de desenvolvimento em que a resposta espectral do trigo, representada pelo IVDN, melhor se relaciona com a fitomassa seca total da parte aérea, quanto para avaliar a variação espectral dos genótipos por meio da análise de agrupamento. Nessa análise, utilizouse, como medida de dissimilaridade, a distância euclidiana e, como método hierárquico aglomerativo, o método de Ward. Por meio desse método, foi estabelecido o dendograma que permitiu verificar o grau de similaridade entre genótipos e grupos similares, ou entre grupos distintos, em termos dos valores médios do fator de reflectância dos genótipos, nas bandas TM1, TM2, TM3 e TM4. As análises de agrupamento foram realizadas utilizando-se o programa STATISTICA for Windows (STATSOFT Inc., 2002).

A eficiência de uso da radiação fotossinteticamente ativa para produção de fitomassa seca total da parte aérea $(\varepsilon F)$, para cada genótipo, foi obtida pela razão entre a fitomassa seca total da parte aérea e a radiação fotossiteticamente ativa absorvida acumulada (RFAAA) ao longo do ciclo da cultura, conforme equação abaixo:

$$
\varepsilon_{\mathrm{F}}=\frac{\text { Produção de fitomassa seca total da parte aérea }\left(\mathrm{g} \mathrm{m}^{-2}\right)}{\operatorname{RFAAA}\left(\mathrm{MJ} \mathrm{m}^{-2}\right)}
$$

Estimou-se a RFAAA pelo somatório dos valores diários da RFA absorvida (RFAA; Equação 3), desde a emergência das plântulas, para o qual foi estipulado o valor de IVDN de 0,15 (solo exposto), até a maturação fisiológica das plantas, quando o IVDN atingiu o valor de 0,5 (RUdorff e BATISTA, 1990). A RFAA foi estimada a partir do produto entre a $f_{\mathrm{A}}$ (Equação 4) e a RFA medida em uma estação meteorológica a poucos metros da área experimental (DAughtry et al., 1992):

$\mathrm{RFAA}_{\text {Diária }}=f_{\mathrm{A}} \cdot \mathrm{RFA}_{\text {Diária }}$

Os valores diários do IVDN (Equação 1), interpolados linearmente entre datas consecutivas de medidas radiométricas, foram utilizados para estimar a $f_{\mathrm{A}}$ conforme a seguinte equação(AsRAR et al., 1984):

$f_{\mathrm{A}}=-0,109+1,253$ IVDN

A colheita do trigo foi realizada na parte central de cada unidade experimental, desprezandose duas linhas de cada lado e $0,5 \mathrm{~m}$ no comprimento, para evitar o efeito de bordadura. $\mathrm{Na}$ análise de variância da RFAAA, $\varepsilon_{F}$ e fitomassa da parte aérea das plantas aplicou-se o teste Duncan $(\mu=0,05)$.

\section{RESULTADOS E DISCUSSÃO}

Nos estádios de florescimento e no início de enchimento de grãos ocorreram os mais altos coeficientes de correlação, da análise entre o IVDN e a produção final de fitomassa seca total da parte aérea (Tabela 1; Figura 1). Considerando que esse índice é coletado remotamente, podendo ser obtido para grandes regiões por meio de imagens de satélites de sensoriamento remoto, o resultado é bastante positivo, concordando com aqueles obtidos por RUDORFF e Batista (1991). Hatfield (1981) também observou os mais altos coeficientes de correlação e a maior variabilidade na resposta espectral de genótipos de trigo, no estádio de enchimento de grão. Outro aspecto favorável na escolha dos estádios de florescimento e do início de enchimento de grãos é que o dossel das plantas está bem desenvolvido e a contribuição dos efeitos do solo, na reflectância, é mínima; além disso, as folhas superiores ainda não amareleceram (BARET et al., 1987).

Com base na distância euclidiana do FR médio nas bandas TM1, TM2, TM3 e TM4, os 20 genótipos de trigo foram distribuídos em três grupos no estádio de florescimento e dois grupos no de enchimento de grãos (Figura 2).

Apesar da aparente dissimilaridade entre os grupos, não foi possível separar esses mesmos grupos quando se utilizou o valor do FR de cada parcela amostral, conforme a fiigura 3, por meio do desviopadrão de cada grupo. 
Tabela 1. Data de coleta de dados radiométricos, estádio fenológico e coeficiente de correlação entre IVDN (índice de vegetação com diferença normalizada) e fitomassa total da parte aérea

\begin{tabular}{lcc}
\hline Data da coleta & Estádio fenológico & $\mathrm{r}$ \\
\hline $16 / 5$ & Emergência das plântulas & 0,49 \\
$25 / 5$ & Planta com 3 folhas & 0,66 \\
$1 . \%$ & Início do perfilhamento & 0,68 \\
$22 / 6$ & Final do emborrachamento & 0,68 \\
$29 / 6$ & Florescimento & 0,73 \\
$14 / 7$ & Enchimento de grãos & 0,79 \\
$25 / 7$ & Grão pastoso & 0,65 \\
$11 / 8$ & Maturação dos grãos & 0,67 \\
\hline
\end{tabular}

A
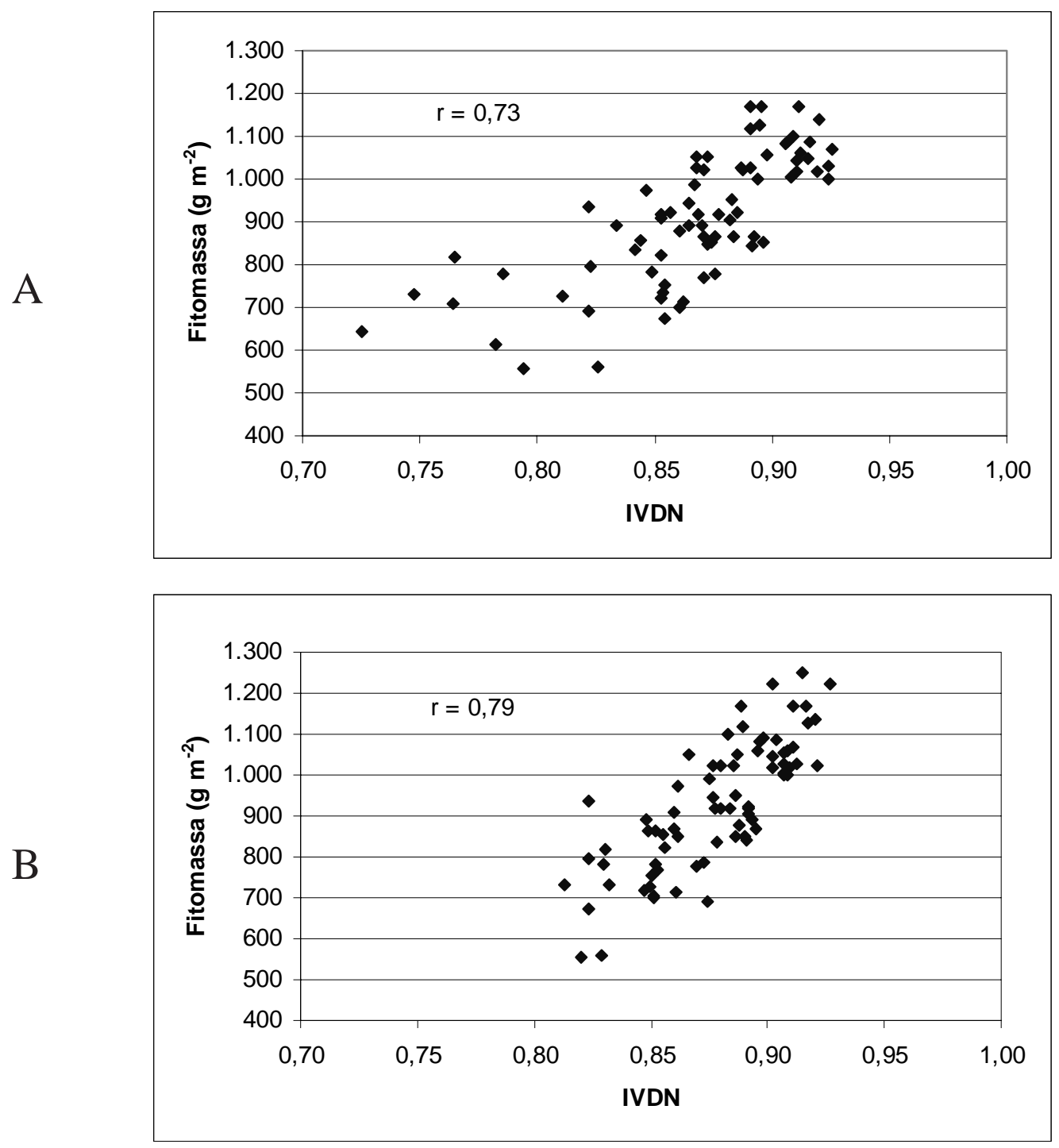

Figura 1. Correlação entre IVDN (índice de vegetação com diferença normalizada) e produção de fitomassa ( $\mathrm{g} \mathrm{m}^{-2}$ ), para os estádios de florescimento (A; 29 de junho) e enchimento de grãos (B; 14 de julho). 
Esse fato ocorre devido à variabilidade na resposta espectral entre parcelas de um mesmo genótipo ser maior do que a variação espectral entre genótipos devido a uma série de fatores, tais como: diferenças no estádio fenológico (AHLRICHS e BAUER, 1983); diferentes condições de vento ao longo da obtenção das medidas radiométricas (MARLOIE, et al., 2001); diferença na posição do radiômetro dentro da parcela; e geometria de iluminação (PINTER JR. et al., 1985).

Pela figura $4 a$, verifica-se que a RFAAA tem uma correlação significativa com a produção de fitomassa seca total da parte aérea $(r=0,78)$. Contudo, a correlação entre RFAAA e $\varepsilon_{\mathrm{F}}$ é bem inferior $(\mathrm{r}=0,44)$ posto que o aumento da radiação solar absorvida está pouco relacionado com a eficiência de conversão da energia solar em química, ou seja, mesmo que a arquitetura do dossel de um genótipo favoreça a absorção da radiação solar incidente, é necessário que a conversão dessa radiação em fotoassimilados seja equivalente. Na tabela 3, nota-se que os valores médios da $\varepsilon_{\mathrm{F}}$ para os genótipos analisados variaram em até $31 \%\left(1,85\right.$ a 2,66 $\left.\mathrm{g} \mathrm{MJ}^{-1}\right)$ enquanto os valores médios de RFAAA variaram em apenas 18\% (353 a $431 \mathrm{MJ} \mathrm{m}^{-2}$ ). A grande variação nos valores de $\varepsilon_{\mathrm{F}}$ contraria a afirmação de que esse valor é relativamente constante para uma dada espécie (Monteith, 1977).

Tabela 2. Agrupamento dos genótipos em função da resposta espectral nas bandas TM1, TM2, TM3 e TM4, para os estádios de florescimento (29 de junho) e enchimento de grãos (14 de julho)

\begin{tabular}{lcr}
\hline $\begin{array}{c}\text { Agrupamento } \\
\text { Espectral }\end{array}$ & Nlorescimento (29 de junho) & Número dos Genótipos* \\
\cline { 2 - 3 } 1 & $2,4,11,12$ & $2,4,6,8,11,12,17,20$ \\
2 & $5,7,9,15,20$ & $1,3,5,7,9,10,13,14,15,16,18,19$ \\
3 & $1,3,6,8,10,13,14,16,17,18,19$ & - \\
\hline
\end{tabular}

* Número do genótipo conforme apresentado na Tabela 3.

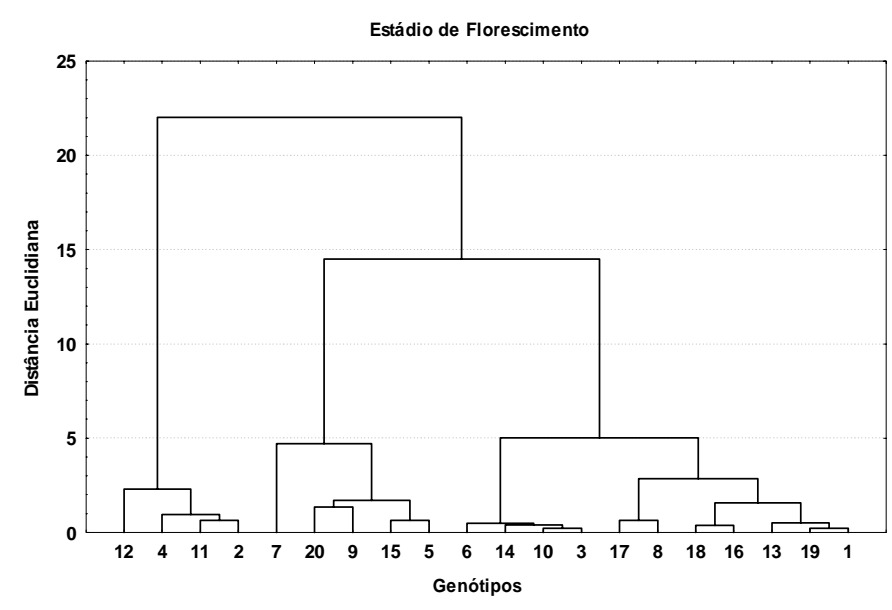

A

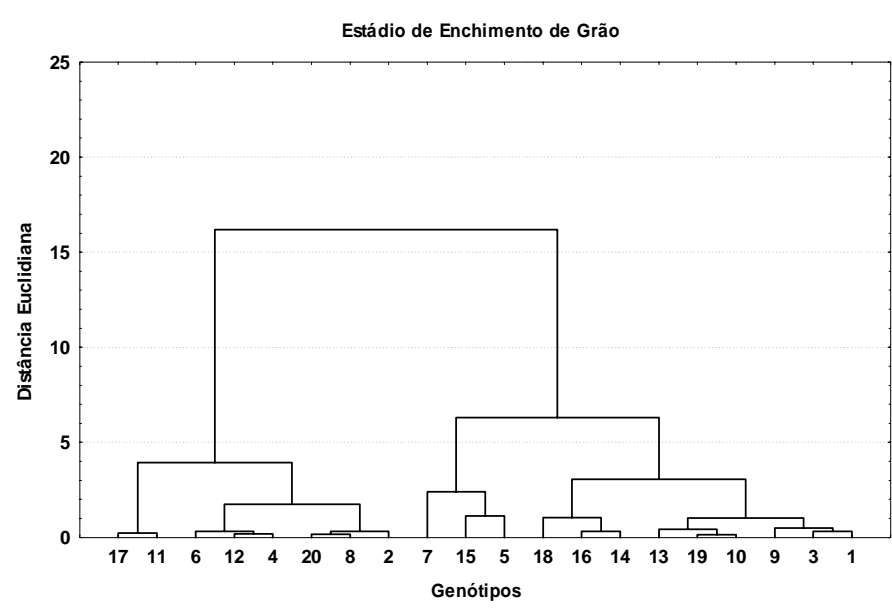

B

Figura 2. Dendograma de similaridade, estabelecido pela análise de agrupamento pelo método hierárquico de Ward, com base na distância euclidiana do FR médio dos genótipos, nas bandas TM1 (450 a 520 nm), TM2 (530 a 610 nm), TM3 (630 a $690 \mathrm{~nm})$ e TM4 (780 a $900 \mathrm{~nm})$, nos estádios fenológicos de florescimento (A) e enchimento de grãos (B). Número dos genótipos conforme Tabela 3. 


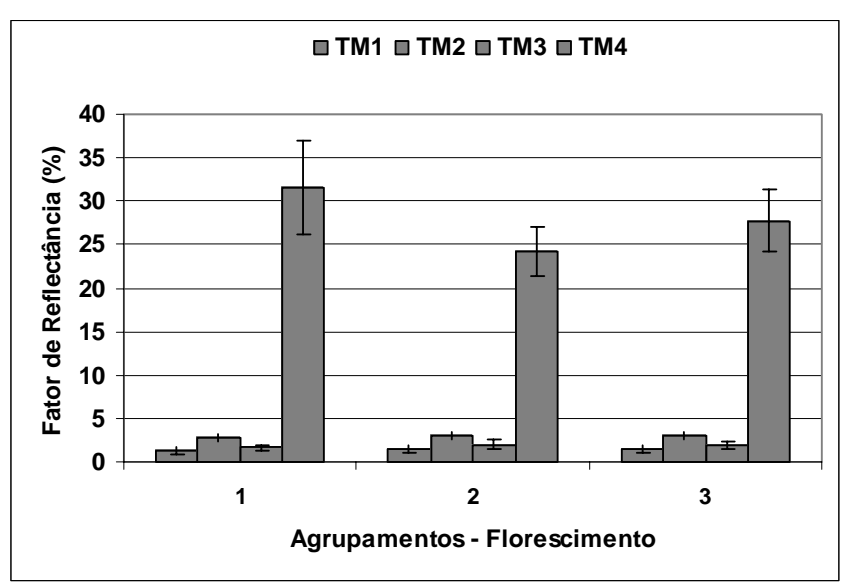

A

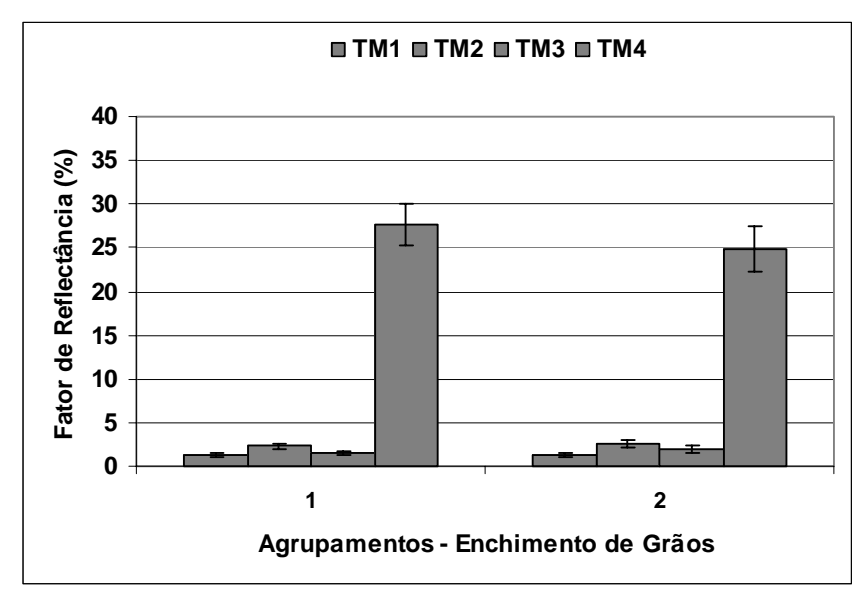

B

Figura 3. Resposta espectral nas bandas TM1 (450 a 520 nm), TM2 (530 a 610 nm), TM3 (630 a 690 nm) e TM4 (780 a 900 $\mathrm{nm}$ ), dos grupos de genótipos de trigo (Tabela 3), nos estádios fenológicos de florescimento (A) e enchimento de grãos (B).

As maiores produções de fitomassa seca não foram observadas para os genótipos com maior capacidade de absorção da radiação, mas para os genótipos com alta eficiência de uso dessa radiação. Por exemplo, os genótipos Kauz² / Chen / / BCN (14) e IAC 373 (20) tiveram a menor e maior eficiência de uso da radiação respectivamente. Contudo, a RFAAA não diferiu significativamente, ao passo que a produção de fitomassa do IAC 373 (20) foi 36,5\% superior ao Kauz*2 / Chen // BCN (14) (Tabela 3). Como exemplo, pode-se observar por meio da comparação entre os genótipos Ana (19) e IAC-373 (20). Em ambos não houve diferença significativa na RFAAA; todavia, a produção de fitomassa difere significativamente, pois a $\varepsilon_{\mathrm{F}}$ do genótipo IAC-373 (20) é significativamente superior ao genótipo Ana (19).
Resultado semelhante pode ser observado por meio da comparação dos genótipos Anahuac/IAC-227 (18) e Kauz*2 / Chen // BCN (14). A correlação entre a $\varepsilon_{\mathrm{F}}$ e a produção de fitomassa seca total da parte aérea é bastante elevada $(r=0,91)$, confirmando que as diferenças na produção de fitomassa estão mais relacionadas com a $\varepsilon_{\mathrm{F}}$ do que com a RFAAA, conforme também observaram CHAPMAN e EDMEADES (1996), em estudo de linhagens de milho (Figura 4b). Portanto, aspectos relacionados à arquitetura da planta, espaçamento entre plantas, disposição das linhas de plantio e outros, que visam aumentar a absorção da RFA incidente, devem considerar também a eficiência com que essa radiação absorvida é convertida em fotoassimilados e direcionada para a produção de grãos.

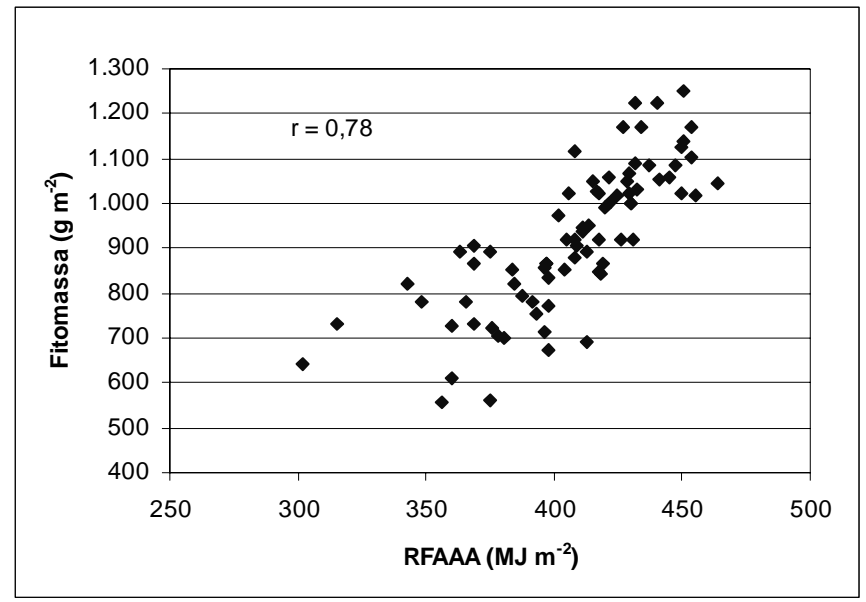

A

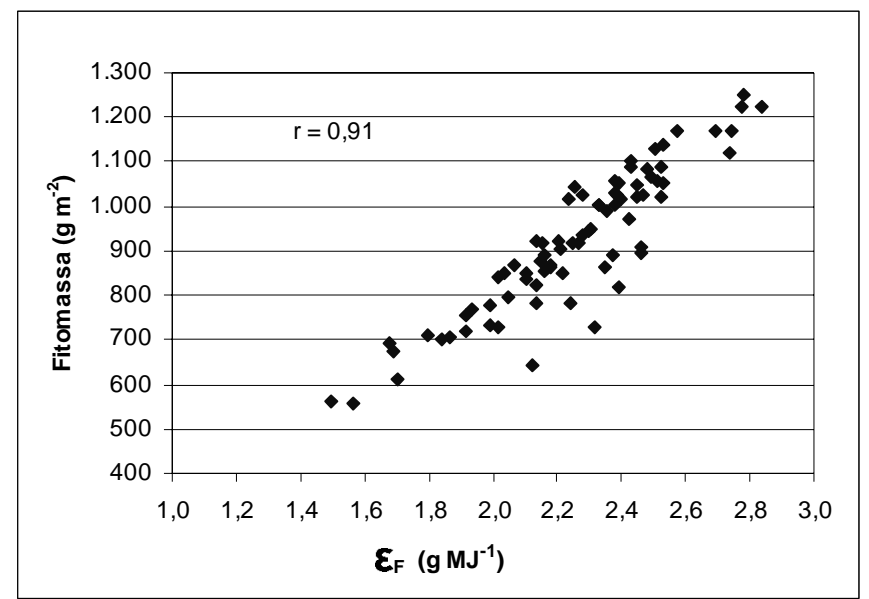

B

Figura 4. Correlação da fitomassa seca total da parte aérea das plantas $\left(\mathrm{g} \mathrm{m}^{-2}\right)$ com: A) Radiação Fotossinteticamente Ativa Absorvida Acumulada (RFAAA; $\mathrm{MJ} \mathrm{m}^{-2}$ ) e B) eficiência de uso da radiação $\left(\varepsilon_{\mathrm{F}} ; \mathrm{g} \mathrm{MJ}^{-1}\right)$. 
Tabela 3. ANOVA dos genótipos para médias de fitomassa total da parte aérea $\left(\mathrm{g} \mathrm{m}^{-2}\right)$, radiação fotossinteticamente ativa absorvida (RFAAA; MJ m²) e eficiência de uso da radiação $\left(\varepsilon_{\mathrm{F}} ; \mathrm{g} \mathrm{MJ}^{-1}\right)$

\begin{tabular}{|c|c|c|c|c|}
\hline $\mathrm{N}^{\circ}$ & Nome do genótipo & Fitomassa & RFAAA & \& \\
\hline 1 & IAC 24 & 931 bcde & 410 abcd & 2,27 cdefgh \\
\hline 2 & IAC 289 & $998 \mathrm{abc}$ & $431 \mathrm{a}$ & 2,32 bcdefg \\
\hline 3 & IAC 315 & 910 bcde & $420 \mathrm{abc}$ & 2,16 efghi \\
\hline 4 & IAC 350 & $1073 \mathrm{ab}$ & $427 \mathrm{abc}$ & $2,51 \mathrm{abc}$ \\
\hline 5 & IAC 351 & 832 cdefg & 370 ef & 2,26 cdefgh \\
\hline 6 & IAC 370 & 974 abcd & 416 abcd & 2,34 abcde \\
\hline 7 & IAC 339 & 796 efg & 391 cde & 2,04 fghij \\
\hline 8 & IAC 364 & $1076 \mathrm{ab}$ & $429 \mathrm{ab}$ & $2,51 \mathrm{abc}$ \\
\hline 9 & Mochis & 962 bcde & $430 \mathrm{a}$ & 2,24 cdefgh \\
\hline 10 & ATTILA & 810 defg & $407 \mathrm{abcd}$ & $1,98 \mathrm{hij}$ \\
\hline 11 & Kauz / CMH77.308 / / Baú & $1003 \mathrm{abc}$ & 417 abcd & 2,39 abcde \\
\hline 12 & Munia // Chen/ Altar 85 & $972 \mathrm{abcd}$ & $425 \mathrm{abc}$ & 2,28 bcdefgh \\
\hline 13 & Chil / 2*Star & $859 \mathrm{cdef}$ & 400 abcde & 2,14 efghi \\
\hline 14 & Kauz $^{* 2}$ / Chen // BCN & $726 \mathrm{fg}$ & 392 bcde & $1,85 \mathrm{j}$ \\
\hline 15 & Kauz *2 / Yaco / / Kauz & $672 \mathrm{~g}$ & $353 \mathrm{f}$ & $1,91 \mathrm{ij}$ \\
\hline 16 & Hahn / Turaco// Turaco & 790 efg & 386 de & 2,03 ghij \\
\hline 17 & Uhu"S" / Seri 82 & $1054 \mathrm{ab}$ & 409 abcd & $2,57 \mathrm{ab}$ \\
\hline 18 & Anahuac/ IAC 227 & 974 abcd & 391 bcde & $2,47 \mathrm{abc}$ \\
\hline 19 & Ana (Tol AL) & 940 bcde & $427 \mathrm{abc}$ & 2,19 defghi \\
\hline 20 & IAC 373 & 1143 a & $423 \mathrm{ab}$ & $2,66 \mathrm{a}$ \\
\hline
\end{tabular}

Médias seguidas pelas mesmas letras não diferem significativamente ao nível de $5 \%$ pelo teste de Duncan.

\section{CONCLUSÃO}

A técnica de radiometria de campo, como ferramenta auxiliar na seleção de genótipos, é promissora na identificação de genótipos com diferentes eficiências de uso da radiação, visto que o aproveitamento da radiação solar absorvida pelas plantas $\left(\varepsilon_{\mathrm{F}}\right)$ é bem mais relevante do que a absorção da radiação solar (RFAAA) na produção de fitomassa, uma vez que a $\varepsilon_{F}$ apresentou significativa variação entre genótipos.

\section{AGRADECIMENTOS}

À Fapesp - Fundação de Amparo à Pesquisa do Estado de São Paulo, pelo financiamento do projeto número 97/11075-7 que possibilitou a realização deste trabalho.

\section{REFERÊNCIAS}

AHLRICHS, J.S.; BAUER, M.E. Relation of agronomic and multispectral reflectance characteristics of spring wheat canopies. Agronomy Journal, Madison, v. 75, p. 987-993, 1983.

ASRAR, G.; FUCHS, M.; KANEMASU, E.T.; HATFIELD, J.L. Estimating absorved photosynthetic radiation and leaf area index from spectral reflectance in wheat. Agronomy Journal, Madison, v.76, n.2, p.300-306, 1984.

BARET, F.; CHAMPION, I.; GUYOT, G.; PODAIRE, A. Monitoring wheat canopies with high spectral resolution radiometer. Remote Sensing of Environment, New York, v. 22, p.367-378, 1987.

CHAPMAN, S.C.; EDMEADES, G.O. Differences in radiation use efficiency among lines in a tropical maize population. In: AUSTRALIAN AGRONOMY CONFERENCE, 8., 1996, Toowoomba. Proceedings... Gosford: The Regional Institute, $1996.5 \mathrm{p}$. 
DEERING, W.D. Field measurements of bidirectional reflectance. In: Theory and Applications of Optical Remote Sensing. New York: Wiley, 1989. p. 14-65.

DAUGHTRY, C.S.T.; GALLO, K.P; GOWARD, S.N.; PRICE, S.D.; KUSTAS, W.P. Spectral estimates of absorbed radiation and phytomass production in corn and soybean canopies. Remote Sensing of Environment, New York, v.39, p.141-152, 1992.

EMBRAPA: Centro Nacional de Pesquisas de Solos (Rio de Janeiro). Sistema brasileiro de classificação de solos. Brasília: Embrapa Produção de Informação; Rio de Janeiro: Embrapa Solos, 1999. 412 p.

GOUDRIAAN, J.; VAN LAAR, H.H. Modelling potential crop growth processes - Textbook with exercises. In: LEFFELAAR, P.A. (Ed.). Current Issues in Production Ecology, Dordrecht, Netherlands: Kluwer Academic Publishers, 1994. v. 2, 238 p.

HATFIELD, J.L. Spectral behavior of wheat yield variety trials. Photogrammetric Engineering and Remote Sensing, London, v.47, p.1487-1491, 1981.

INSTITUTO AGRONÔMICO (IAC). Trigo: recomendações. 2.ed. Campinas: IAC, 1999. 100p. (Boletim Técnico, 167).

MILTON, E.J. Principles of field spectroscopy. International Journal of Remote Sensing, Basingstoke, v.8, n.12, p. 18071827, 1987.

MARLOIE, O.; KÖTZ, B.; BRUGUIER, N.; BARET, F. Influence of the wind on canopy reflectance. The case of maize and wheat crops. In: SYMPOSIUM ON PHYSICAL MEASUREMENTS AND SIGNATURES IN REMOTE SENSING, 8., 2001, Aussois.
Proceedings... ISPRS, 2001.6p.

MONTEITH, J.L. Climate and the efficiency of crop production in Britain. Philosophical Transactions of the Royal Society, London, B281, p. 277-294, 1977.

PINTER JR., P.J.; JACKSON, R.D.; EZRA, C.E.; GAUSMAN, H.W. Sun-angle and canopy-architecture effects on the spectral reflectance of six wheat cultivars. International Journal of Remote Sensing, Basingstoke, v.6, n.12, p.1813-25, 1985.

RUDORFF, B.F.T.; BATISTA, G.T. Spectral response of wheat and its relationship with agronomic variables in the tropical region. Remote Sensing of Environment, New York, v.31, p.53-63, 1990.

RUDORFF, B.F.T.; BATISTA, G.T. Wheat yield estimation at the farm level using Landsat TM and agrometeorological data. International Journal of Remote Sensing, Basingstoke, v.12, p. 2477-2484, 1991.

STEFFEN, C.A. Técnicas radiométricas com o Spectron SE590. In: SIMPÓSIO BRASILEIRO DE SENSORIAMENTO REMOTO, 7., 1993, Curitiba. Anais... Curitiba: INPE/SELPER, 1993. 7p.

STATSOFT INC. Statistica for Windows: version 2.0. Tulsa, Estados Unidos, 2002.

TUCKER, C.J. Red and photographic infrared linear combination for monitoring vegetation. Remote Sensing of Environment, New York, v. 8, n.2, p.127-150, 1979. 